\title{
Inverse Emulsion Polymerization of Acrylamide Initiated by Oil- and Water-soluble Initiators: Effect of Emulsifier Concentration
}

\author{
Ignác CAPEK ${ }^{\dagger}$ \\ Polymer Institute, Slovak Academy of Sciences, Dúbravská cesta 9, 84236 Bratislava, Slovakia
}

(Received February 16, 2004; Accepted June 8, 2004; Published October 15, 2004)

\begin{abstract}
The nature of the reaction loci in inverse emulsion polymerization of acrylamide (AAm) initiated by oil (2,2'-azoisobutyronitrile, AIBN)- and water-soluble (ammonium peroxodisulfate, APS) initiators was investigated. The rate of polymerization $\left(R_{\mathrm{p}, \max }\right.$, Interval 2$)$ increases with increasing the emulsifier concentration up to a certain critical concentration and then decreases. The rate of polymerization and the polymerization rate per particle are somewhat larger in the APS-initiated polymerization than in the AIBN-initiated one. For both initiators the polymerization rate per particle is proportional to the particle size and decreases with increasing the emulsifier concentration and the decrease is much more pronounced at the high emulsifier concentrations. The similar kinetic dependences for both initiators were discussed in terms of the formation of monomeric or oligomeric radicals with the similar nature, mobility via the reaction system and partitioning between the oil and water phases. The similar radicals carrying the initiator fragment were formed by the addition of one or two (or several) units to the primary radicals $\left(\mathrm{SO}_{4}{ }^{\bullet-}\right.$ or 2-cyanoisopropyl). The estimated average number of radicals per particle $(\bar{n})$ is much below 0.5 . The low radical concentration in the polymer particles was discussed in terms of the desorption of monomeric and emulsifier radicals. The desorption of radicals was confirmed by both the Nomura model $\left(N_{\text {model }}, k_{\text {des }, N}^{\prime}\right)$ and the Ugelstad approach (the solution of population balance for the particle with $\bar{n}, T_{\text {model }}, k_{\text {des }, T}^{\prime}$ ). The $k_{\text {des }, T}^{\prime}$ 's were found to be much larger than the $k_{\text {des }, N}^{\prime}$ 's. The differences in the $k_{\mathrm{des}}^{\prime}$ 's were discussed in terms of the desorption (surface active) monomeric radicals (the polymerization within the outer spheres of polymer particles) and emulsifier (the chain transfer to emulsifier) radicals. The radical entry rate decreases with increasing the emulsifier concentration or decreasing the particle size.

[DOI 10.1295/polymj.36.793]

KEY WORDS Radical Polymerization / Inverse Emulsion / Acrylamide / Absorption and Exit of Radicals / Tween 85 /
\end{abstract}

With the increased interest in the technological exploitation of inverse emulsion polymerization as a method for the preparation of water soluble polymers has come the need to understand microscopic kinetic events that govern both the particle nucleation and the polymer growth in the polymer particles. The conventional processes such the initiation, propagation, termination and chain transfer are all operative in the inverse emulsion or microemulsion polymerizations. The inverse microemulsion polymerization processes (water-in-oil systems, w/o) are well known. ${ }^{1-4}$ The polymerization is very fast and the process is described by two non-stationary rate intervals. This is ascribed to two opposing effects; the continual increase of particle number and continual decrease of monomer at the reaction loci. The microdroplets saturated with the aqueous solution of monomer act as reaction loci and the reservoir of monomer. The rate of inverse microemulsion polymerization increases with increasing the initiator concentration but strongly decreases with increasing the emulsifier concentration. The study devoted to the inverse miniemulsion (homogenized emulsion) polymerization led to the conclusion that the addition of lipophobe to the inverse miniemulsion generated the long term stability against Ostwald ripening. ${ }^{5}$ The inverse emulsion polymerization of acrylamide produced turbid, viscous and unstable dispersions at the outset and during the intermediate stages of the polymerization. ${ }^{6-8}$ It was observed that the initial rate of inverse emulsion polymerization is first order with respect to the monomer concentration, first order with the initiator concentration and zeroth order with respect to the (nonionic) emulsifier concentration. The maximal polymerization rate shifts to the higher conversion with increasing temperature but to the lower conversion with increasing the acrylamide concentration and oil phase level. The number of polymer particles and the particle size slightly increased with increasing conversion. Increasing the emulsifier concentration, the inverse emulsion was transformed to the transparent inverse lattices. 
The mechanism that lead to the generation of primary radicals in the $\mathrm{o} / \mathrm{w}$ emulsion polymerization initiated by oil-soluble initiators are divided into two approaches: 1) those in which radicals are formed by desorption of radicals from the polymer particles ${ }^{9}$ and 2) those that considered that the radicals formed in the aqueous phase from the fraction of oil-soluble initiator dissolved in the aqueous phase are responsible for the kinetic similarities between oil-soluble and water-soluble initiators. ${ }^{10,11}$ Asua et al. ${ }^{12}$ suggested that the single radicals derived from the oil-soluble initiator exit to the aqueous phase and take part in termination. The similar mechanism of the primary radical generation can be expected for the inversion emulsion polymerization of acrylamide initiated by the water-soluble initiator. In the present paper, the experimental design based on the inverse emulsion polymerizations initiated by both the oil-soluble and water-soluble initiators was envisaged in an attempt to elucidate the main locus of radical formation. The solubility of radicals and the partitioning of radicals between the oil- and water phases is suggested to be a dominant parameter of the polymerization process. Thus, if the single radicals were formed in the continuous phase then the hydrophobic radicals derived from the 2,2'-azoisobutyronitrile (AIBN) could not enter the hydrophilic polymer particles. The similar behavior one can expect for the primary radicals derived from ammonium peroxodisulfate (APS) formed in the disperse aqueous phase. The transformation of hydrophobic (AIBN) radicals to the surface active or more hydrophilic ones by the continuous phase polymerization enables them enter the hydrophilic particle. The similar behavior one can be expected for the hydrophilic (APS) radicals, that is, the addition of one or two monomer units to the hydrophilic radicals forms less hydrophilic oligomeric radicals able to desorb from the polymer particles to the continuous phase and then re-enter the micelles or particles as well. Under such conditions the rate of polymerization would be less sensitive or even independent of the initiator nature. The variation in the emulsifier concentration is expected to vary the partitioning of radicals between the oil and water phases.

According to our best knowledge there are no studies on the desorption of monomeric radicals in the inverse microemulsion and emulsion polymerizations of acrylamide. The acrylamide monomer was reported to increase the colloidal stability of monomer and polymer dispersion and have a favorable effect on the whole polymerization process. Acrylamide monomer acts as a co-emulsifier and is partly located between the paraffinic chain of the emulsifier at the water/oil interface and partly inside the water pool of the inverse micelles. ${ }^{2}$ The contribution of the polymeriza- tion in the interfacial layer is open for the experimental verification in the inverse emulsion polymerization of acrylamide. Furthermore, this paper is also devoted to the desorption of monomeric radicals modeled by the Nomura model $\left(N_{\text {model }}\right)^{13}$ and Ugelstad approach. ${ }^{14}$ Both approaches are applicable for the emulsion polymerization where the initiating radicals are generated by the decomposition of initiator dissolved in the continuous phase. The inverse AIBN-initiated polymerization of acrylamide, thus, fulfills this condition. The Ugelstad approach is based on the solution of the population balance for the polymer particles with $\bar{n}$.

\section{EXPERIMENTAL}

\section{Materials}

Acrylamide (AAm) (anal. grade, from Fluka) was used without further purification. Extra pure ammonium peroxodisulfate (APS, Fluka) was used as supplied. 2,2'-Azoisobutyronitrile (AIBN) was recrystallized from ethanol. The emulsifier used was the reagent-grade Tween 85 (non-ionic emulsifier, polyoxyethylene sorbitan trioleate, provided by Serva, Tw 85). Doubly distilled water used for the preparation of emulsion was deprived of oxygen by heating to boiling point and cooling under a stream of argon.

\section{Recipe and Procedures}

Batch inverse emulsion polymerizations of unsaturated monomers (AAm) were carried out at $60^{\circ} \mathrm{C}$. The recipe comprising $100 \mathrm{~g}$ cyclohexane, $35 \mathrm{~g}$ water, $5 \mathrm{~g}$ AAm and $0.104 \mathrm{~g}$ APS or $0.2108 \mathrm{~g}$ AIBN was used. The amount of Tw 85 varied as shown later. The inverse emulsion polymerization of AAm is very fast and therefore the use of gravimetry was limited because it provides the data in the high conversion interval. The low and medium conversion intervals can be obtained by the dilatometry which, however, requires the stable monomer dispersion. This was reason why the monomer emulsion was homogenized before it could be used for the dilatometric measurements. Furthermore, the small amount of reaction mixture and the very thin reaction vessel were used to depress the delayed heating of the sample (to the reaction temperature) due to the very fast polymerization. The homogenized inverse-monomer dispersions with a higher stability were prepared by the homogenizer Ultra Turrax, IKA Works, USA. The final conversion of several samples was also checked by the gravimetry. The contraction factor (CF) of acrylamide was estimated to be 0.3 which is in a good agreement with the literature values. ${ }^{2,4}$ The particle size was determined by the dynamic light scattering method. The polymerization technique, the preparation of polymer 
Table I. Variation of kinetic and colloidal parameters in the inverse emulsion polymerization of AAm initiated by AIBN with the Tw 85 concentration $^{\text {a }}$

\begin{tabular}{cccccc}
\hline $\begin{array}{c}\text { Tw 85] } \times 10^{2} \\
\left(\mathrm{~mol} \mathrm{dm}^{-3}\right)\end{array}$ & $\begin{array}{c}R_{\mathrm{p}, \max } \times 10^{4} \\
\left(\mathrm{~mol} \mathrm{dm}^{-3} \mathrm{~s}^{-1}\right)\end{array}$ & $\begin{array}{c}R_{\mathrm{pp}} \times 10^{20} \\
\left(\mathrm{~mol} \mathrm{~s}^{-1} \mathrm{particle}^{-1}\right)\end{array}$ & $\begin{array}{c}d_{\mathrm{p}} \\
(\mathrm{nm})\end{array}$ & $\begin{array}{c}N_{\mathrm{p}} \times 10^{-16} \\
\left(\mathrm{dm}^{3}\right)\end{array}$ & $\begin{array}{c}\bar{n} \times 10^{2} \\
(\text { particle })\end{array}$ \\
\hline 2.72 & 9.0 & 4.5 & 200 & 2.0 & 3.7 \\
5.44 & 13.7 & 3.6 & 162 & 3.8 & 3.0 \\
8.16 & 14.4 & 3.1 & 152 & 4.6 & 2.6 \\
10.88 & 11.7 & 1.7 & 134 & 6.8 & 1.4 \\
13.6 & 10.6 & 1.2 & 124 & 8.5 & 1.1 \\
\hline
\end{tabular}

${ }^{\mathrm{a}} 100 \mathrm{~g}$ cyclohexane, $35 \mathrm{~g}$ water, $5 \mathrm{~g}$ AAm, $0.2108 \mathrm{~g}$ AIBN $\left\{[\mathrm{AIBN}]_{\mathrm{CH}}=0.0096 \mathrm{~mol} \mathrm{dm}^{-3}\right.$-related to the cyclohexane phase, $\left([\mathrm{AIBN}]_{\mathrm{tot}}=0.0076 \mathrm{~mol} \mathrm{dm}^{-3}=\right.$ related to the whole reaction volume (water plus cyclohexane) $\}, 60^{\circ} \mathrm{C}$. [Tw 85], $R_{\mathrm{p}, \max }$ and $N_{\mathrm{p}}$ are related to the continuous phase (cyclohexane).

Table II. Variation of kinetic and colloidal parameters in the inverse emulsion polymerization of AAm initiated by APS with the Tw 85 concentration $^{\mathrm{a}}$

\begin{tabular}{cccccc}
\hline $\begin{array}{c}\text { Tw 85] } \times 10^{2} \\
\left(\mathrm{~mol} \mathrm{dm}^{-3}\right)\end{array}$ & $\begin{array}{c}R_{\mathrm{p}, \max } \times 10^{4} \\
\left(\mathrm{~mol} \mathrm{dm}^{-3} \mathrm{~s}^{-1}\right)\end{array}$ & $\begin{array}{c}R_{\mathrm{pp}} \times 10^{20} \\
\left(\mathrm{~mol} \mathrm{~s}^{-1} \mathrm{particle}^{-1}\right)\end{array}$ & $\begin{array}{c}d_{\mathrm{p}} \\
(\mathrm{nm})\end{array}$ & $\begin{array}{c}N_{\mathrm{p}} \times 10^{-16} \\
\left(\mathrm{dm}^{3}\right)\end{array}$ & $\begin{array}{c}\bar{n} \times 10^{2} \\
(\text { particle })\end{array}$ \\
\hline 2.72 & 15.0 & 16.9 & 265 & 0.89 & 13.8 \\
5.44 & 20.6 & 9.4 & 193 & 2.2 & 7.7 \\
8.16 & 26.2 & 3.7 & 130 & 7.2 & 3.0 \\
10.88 & 29.7 & 2.4 & 110 & 12.2 & 2.0 \\
13.6 & 26.6 & 0.8 & 80 & 31.7 & 0.7 \\
\hline
\end{tabular}

${ }^{\mathrm{a}} 100 \mathrm{~g}$ cyclohexane, $35 \mathrm{~g}$ water, $5 \mathrm{~g} \mathrm{AAm}, 0.104 \mathrm{~g}$ APS $\left\{[\mathrm{APS}]_{\mathrm{aq}}=0.013 \mathrm{~mol} \mathrm{dm}^{-3}\right.$-related to the aqueous phase, ([APS $]_{\text {tot }}=0.0027 \mathrm{~mol} \mathrm{dm}^{-3}=$ related to the whole reaction volume (water plus cyclohexane) $\}, 60^{\circ} \mathrm{C}$. [Tw 85$]$, $R_{\mathrm{p}, \max }$ and $N_{\mathrm{p}}$ are related to the continuous phase.

latex for size measurements, the estimation of particle number were the same as described earlier. ${ }^{15}$ Limiting viscosity numbers $[\eta]$ of polyacrylamide were determined with Ubbelohde viscometer in water at $30^{\circ} \mathrm{C}$. The viscosity-average molecular weight, $M_{\mathrm{v}}$, of polyacrylamide was calculated from $[\eta]=6.31 \times 10^{-5}$ $M_{\mathrm{v}}{ }^{0.8} \cdot{ }^{16}$

The homogenized inverse emulsion prepared was used for Ostwald ripening measurements. The colloidal stability of the inverse (non-diluted) emulsion was monitored by placing about $100 \mathrm{~mL}$ sample in a glass vial at $25^{\circ} \mathrm{C}$. The position of the creaming line from the bottom of the sample or the time necessary for a visible phase to appear was then recorded.

\section{Spectral Measurements}

In order to determine the solubility of AIBN in the aqueous phase or the partitioning of AIBN between water (the disperse phase) and cyclohexane (the continuous phase), the absorbance of the oil and aqueous phases saturated with AIBN was measured at $25^{\circ} \mathrm{C}$. After dissolving of $0.2108 \mathrm{~g}$ of AIBN in $134 \mathrm{~mL}$ of cyclohexane $(\mathrm{CH}), 35 \mathrm{~g}$ of water was added. Then the water/AIBN/CH mixture was stirred at $400 \mathrm{rpm}$ for ca. $24 \mathrm{~h}$ at $25^{\circ} \mathrm{C}$. After standing the mixture for several hours at $25^{\circ} \mathrm{C}$, the separated cyclohexane and aqueous layers were used for spectroscopic measurements.

\section{RESULTS AND DISCUSSION}

The Phase Behavior and Monomer Droplet Degradation

Inverse emulsions based on acrylamide (AAm) were prepared by mild mixing a cyclohexane $(\mathrm{CH})$ solution of Tween 85 with a water solution of AAm at a room temperature. The prepared monomer dispersions were unstable and separated into two distinct phases after ceasing of stirring. When the dispersion was treated by vigorous mixing (see Experimental section) the stability of the monomer dispersion abruptly increased. Under the latter condition the inverse AAm/water/Tween $85 / \mathrm{CH}$ emulsions (recipes: Tables I and II) were stable for several days at a room temperature. The homogenization of water/cyclohexane/emulsifier mixtures (the emulsifier concentrations used in the polymerization recipes were much above the critical micelle concentration (CMC) of Tween $85, \mathrm{CMC}=1 \times 10^{-3} \mathrm{~mol} / \mathrm{dm}^{-3}$ in cyclohexane at $25^{\circ} \mathrm{C}^{17}$ ) produced the stable fine monomer emulsion.

\section{Polymerization Rate}

The effect of the emulsifier ( $\mathrm{Tw} 85$ ) concentration on the AIBN-initiated emulsion polymerization of acrylamide (AAm) is summarized in Figure 1. In this 


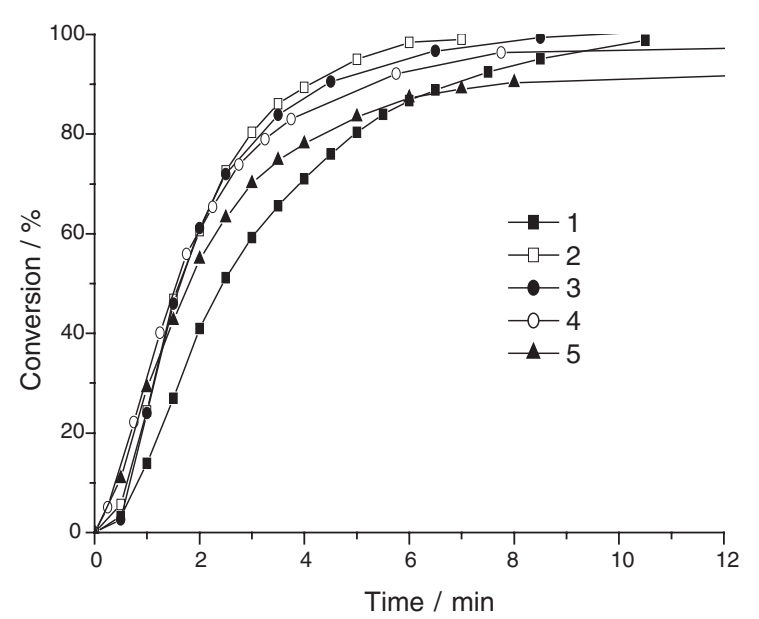

Figure 1. Variation of monomer conversion in the inverse emulsion polymerization of acrylamide (AAm) with Tween 85 (Tw 85) concentration and reaction time. Recipe: $100 \mathrm{~g}$ cyclohexane, $35 \mathrm{~g}$ water, $5 \mathrm{~g}$ AAm, $0.2108 \mathrm{~g}$ AIBN, $60^{\circ} \mathrm{C}$. [Tw 85] $\times 10^{2} /$ (mol dm ${ }^{-3} \mathrm{CH}$-related to cyclohexane): (1) 2.72 , (2) 5.44 , (3) 8.16, (4) 10.88 , (5) 13.6.

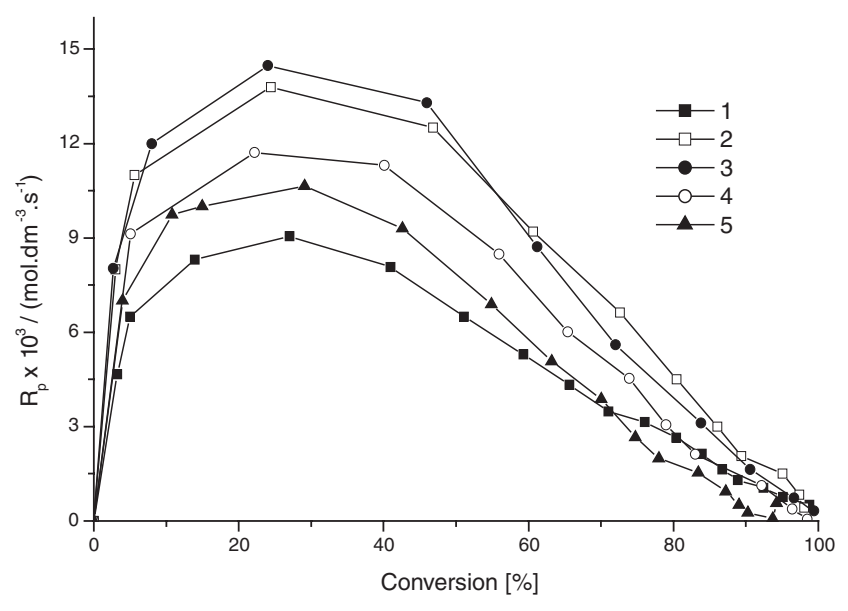

Figure 2. Variation of the rate of polymerization in the inverse emulsion polymerization of AAm with the Tw 85 concentration and conversion. Recipe: $100 \mathrm{~g}$ cyclohexane, $35 \mathrm{~g}$ water, $5 \mathrm{~g}$ AAm $\left(=0.703 \mathrm{~mol} \mathrm{dm}^{-3}\right.$ water $), 0.2108 \mathrm{~g} \mathrm{AIBN}, 60^{\circ} \mathrm{C}$. [Tw $85] \times 10^{2} /\left(\mathrm{mol} \mathrm{dm}^{-3} \mathrm{CH}\right):$ (1) 2.72 , (2) 5.44 , (3) 8.16 , (4) 10.88, (5) 13.6.

Table III. Kinetic parameters for inverse emulsion polymerization of AAm

\begin{tabular}{lll}
\hline Parameter & \multicolumn{1}{c}{ Numerical value } & Ref. \\
\hline$[\mathrm{AAm}]_{\mathrm{p}}$ & $1.2 \mathrm{~mol} \mathrm{dm}^{-3}$ & - \\
$k_{\mathrm{d}, \mathrm{APS}}$ & $6.06 \times 10^{16}(-140167 / R T)$ & 18 \\
$k_{\mathrm{d}, \mathrm{AIBN}}$ & $7.0 \times 10^{16}(-139805 / R T)$ & 19 \\
$F$ & 0.5 & 18 \\
$k_{\mathrm{p}}$ & $9.9 \times 10^{7} \exp (-2743 / R T) \mathrm{dm}^{3} \mathrm{~mol}^{-1} \mathrm{~min}^{-1}$ & 20,21 \\
$k_{\mathrm{t}}$ & $5.07 \times 10^{11} \exp (-1482 / R T) \mathrm{dm}^{3} \mathrm{~mol}^{-1} \mathrm{~min}^{-1}$ & $20-22$ \\
$k_{\mathrm{fm}}$ & $5.73 \times 10^{8} \mathrm{exp}(-10438 / R T) \mathrm{dm}^{3} \mathrm{~mol}^{-1} \mathrm{~min}^{-1}$ & $20-22$ \\
$m_{\mathrm{d}}$ & $0.093(\mathrm{styrene} /$ water or benzene $/$ water $)$ & 23,24 \\
$D_{\mathrm{c}}$ & $2.933 \times 10^{-9} \mathrm{~cm}^{2} / \mathrm{s}$ & 25 \\
$D_{\mathrm{p}}$ & $2.933 \times 10^{-12} \mathrm{~cm}^{2} / \mathrm{s}$ & 25 \\
\hline
\end{tabular}

case the oil soluble initiator (AIBN) is dissolved in the continuous organic phase. The initiating (2-cyanoisopropyl) radicals are formed by the decomposition of hydrophobic AIBN. According to the micellar model the hydrophobic (AIBN fragments-2-cyanoisopropyl) radicals cannot enter the hydrophilic polymer particles. This can be avoided by the continuous phase polymerization of acrylamide forming the surface active or more hydrophilic radicals which can be adsorbed by the hydrophilic polymer particles. The polymerization is very fast and the final conversion (close to ca. $100 \%$ ) is reached in ca. $10 \mathrm{~min}$. The fast polymerization can be attributed to the high entry rate efficiency of oligomeric radicals into the polymer particles, the very high propagation rate constant of AAm (Table III) and the compartmentalization of the reaction loci. The dependence of the rate of polymerization vs. conversion can be described (for all runs) by a curve with the three rate intervals (Figure 2). ${ }^{26}$ The rate of polymerization $\left(R_{\mathrm{p}}\right)$ at first abruptly in- creases to the maximum $\left(R_{\mathrm{p}, \max }\right)$, then keeps a constant value up to $c a$. $50 \%$ conversion and finally decreases up to the final conversion. The abrupt increase in the initial rate of polymerization can result from the robust particle nucleation. The rate of polymerization first increases with increasing the emulsifier concentration (Figure 2, the curves 1, 2 and 3) and then decreases (the curve 4 is below the curve 3 and the curve 5 is below the curve 4 ).

In the AIBN-initiated polymerization the dependence of the maximal rate of polymerization (estimated from curves 1-5, Figure 2) vs. Tw 85 concentration is described by a curve with a maximum at $c a$. [Tw $85]=0.08-0.09 \mathrm{~mol} \mathrm{dm}^{-3}$ (Figure 3 , curve 2$)$. The increase in the polymerization rate (given by the curves 1-3, Figure 2) can be attributed to the increase of the number of particles with increasing the emulsifier concentration (Table I). The decrease in the polymerization rate appears at the higher emulsifier concentration which deviates from the micellar model. ${ }^{27}$ 


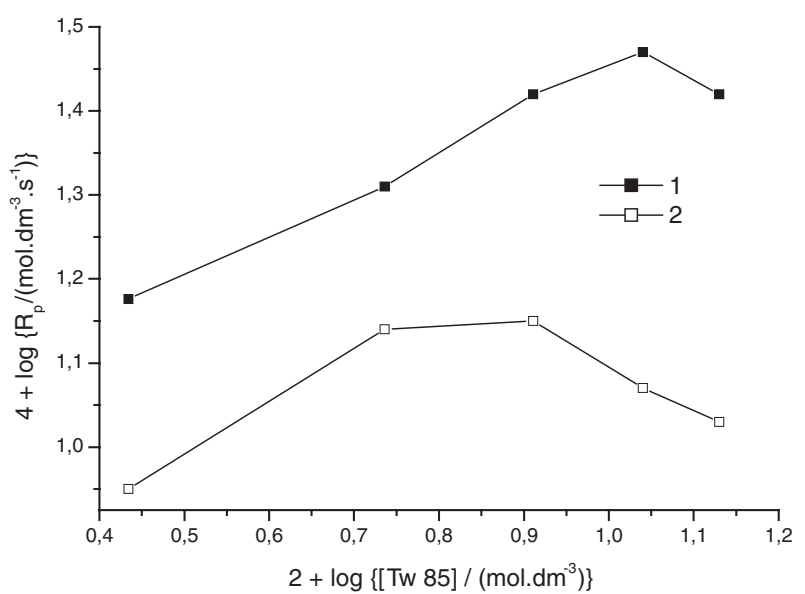

Figure 3. Variation of the rate of polymerization in the inverse emulsion polymerization of AAm with the Tw 85 concentration and initiator type. (1): APS, (2): AIBN. Other conditions see in the legends to Figures 2 and 6.

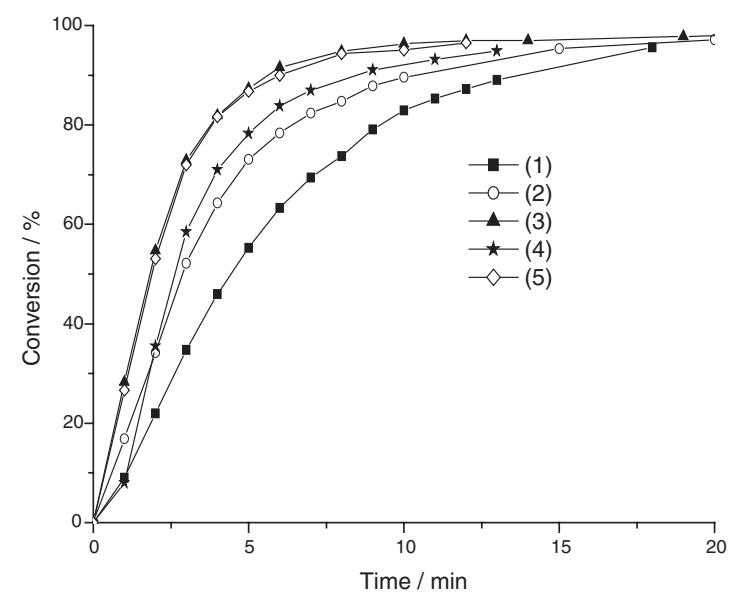

Figure 4. Variation of monomer conversion in the inverse emulsion polymerization of AAm with Tw 85 concentration and reaction time. Recipe: $100 \mathrm{~g}$ cyclohexane, $35 \mathrm{~g}$ water, $5 \mathrm{~g}$ AAm, $0.104 \mathrm{~g}$ APS, $60^{\circ} \mathrm{C}$. [Tw 85] $\times 10^{2} /\left(\mathrm{mol} \mathrm{dm}^{-3} \mathrm{CH}\right)$ : (1) 2.72 , (2) 5.44, (3) 8.16 , (4) 10.88 , (5) 13.6.

Figure 4 shows that the polymerization rate per particle strongly decreases at the higher emulsifier concentration. The strong decrease in the polymerization rate with increasing the emulsifier concentration was observed in the inverse microemulsion polymerization of AAm $\left(R_{\mathrm{p}, \max } v s\right.$. [emulsifier] $\left.{ }^{x=-0.55}\right) .^{28,29}$ The deviation from the micellar model, thus, can be discussed in terms of the decreased monomer concentration at the particle loci (diluted by the emulsifier molecules) and the increased desorption of radicals (monomeric and emulsifier) from particles (typical for the small polymer particles).

In the APS-initiated emulsion polymerization the primary radicals derived from the APS initiator are dissolved in the aqueous (disperse) phase where they

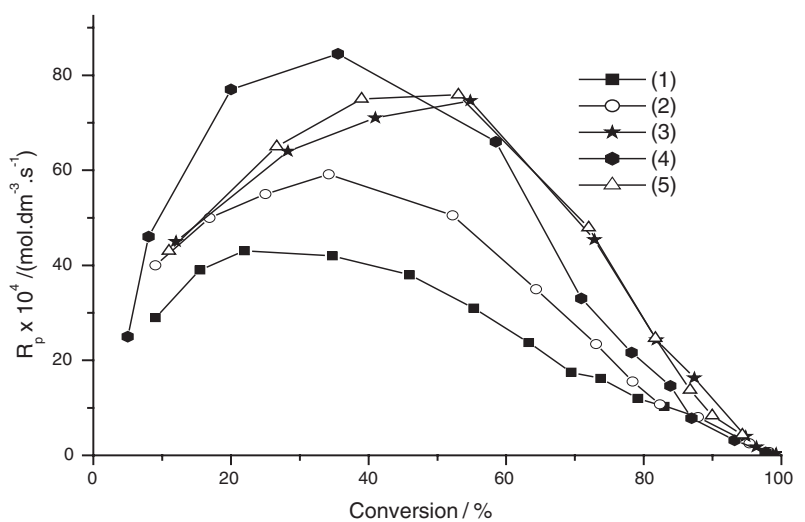

Figure 5. Variation of the rate of polymerization in the inverse emulsion polymerization of AAm with the Tw 85 concentration and conversion. Recipe: $100 \mathrm{~g}$ cyclohexane, $35 \mathrm{~g}$ water, $5 \mathrm{~g}$ AAm, $0.104 \mathrm{~g}$ APS, $60^{\circ} \mathrm{C}$. [Tw 85] $\times 10^{2} /\left(\mathrm{mol} \mathrm{dm}^{-3} \mathrm{CH}\right)$ : (1) 2.72 , (2) 5.44, (3) 8.16 , (4) 10.88, , (5) 13.6.

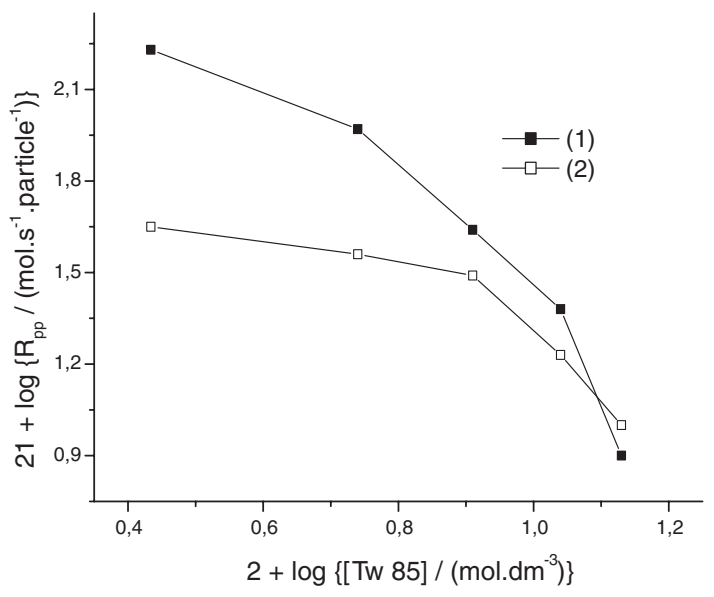

Figure 6. Variation of the polymerization rate per particle in the inverse emulsion polymerization of AAm with the Tw 85 concentration and initiator type. (1): APS, (2): AIBN. Other conditions see in the legends to Figures 2 and 5 and Tables I and II.

start the polymerization. The cage effect or the recombination of primary radicals $\left(\mathrm{SO}_{4}{ }^{--}\right)$, however, strongly disfavors the initiation polymerization. The addition of one or more monomer units to the primary $\left(\mathrm{SO}_{4}{ }^{\bullet-}\right)$ radicals can generate the radicals able to exit from the cage effect or polymer particle and start the polymerization. The conversion-time data and the polymerization rate-conversion data for the inverse emulsion polymerization of acrylamide initiated by APS shown in Figures 5 and 6 result from the efficient formation of initiating radicals. The shape of conversion and polymerization rate curves is very similar to those for the AIBN-initiated polymerization (see above).

Figure 6 shows that the maximal rate of APS-initiated polymerization (the curve 1) increases with in- 
creasing the emulsifier concentration. The maximal rate of polymerization appears at $[\mathrm{Tw} 85]=0.11$ mol dm ${ }^{-3}$ (Figure 3, curve 1) and at $[\mathrm{Tw} 85]=0.08$ mol dm ${ }^{-3}$ in the APS-initiated polymerization or in the AIBN-initiated one (see above), respectively. The increase in the polymerization rate with increasing the emulsifier concentration (Figure 5, curves 1$4)$. The reaction parameter $x=0.5$ on the emulsifier concentration $\left(R_{\mathrm{p}, \max } \propto[\mathrm{Tw} 85]^{x}\right.$, up to $[\mathrm{Tw} 85]=$ $0.11 \mathrm{~mol} \mathrm{dm}^{-3}$, from Figure 5, curves 1-4) is in a good agreement with the classical micellar models $(x=0.6){ }^{27,30}$ The parameter $x=0.5$ indicates that the emulsifier acts as the stabilizer and generator of polymer particles as well. The decrease in the polymerization rate at the highest emulsifier concentration (Figure 5, curves 4 and 5) deviates from the micellar model. ${ }^{27}$ The similar trend was observed in the polymerization rate per particle $v s$. the emulsifier concentration (Figure 4). The decrease in the polymerization rate at a higher emulsifier concentration is attributed to the decreased radical concentration in the polymer particle. The dilution of monomer concentration with the emulsifier molecules is further parameter which depresses the polymerization rate.

At low emulsifier concentrations the polymerization rate per polymer particle $\left(R_{\mathrm{pp}}\right)$ is larger for the APSinitiated polymerization than for the AIBN-initiated polymerization (Figure 4). The difference between the $R_{\mathrm{pp}}$ values continuously decreases with increasing the emulsifier concentration and the $R_{\mathrm{pp}}$ 's are nearly same at high concentrations of emulsifier. When we relate the initiator concentration to the whole reaction volume (cyclohexane plus water) then the molar ratio both initiators $[\mathrm{AIBN}]_{\text {tot }} /[\mathrm{APS}]_{\text {tot }}$ is 2.8 (Tables I and II). Under the free diffusion of initiator molecules via both phases and the ratio $[\mathrm{AIBN}]_{\mathrm{tot}} /[\mathrm{APS}]_{\mathrm{tot}}=2.8$ the polymerization would be faster in the AIBN-initiated system. The polymerization rate shows that the reverse is true, that is, the APS-initiated polymerization is faster than the AIBN-initiated one. In the second limiting approach the initiator dissolves and forms the initiating radicals in one phase (AIBN in cyclohexane and APS in water) the molar ratio $[\mathrm{AIBN}]_{\mathrm{CH}} /[\mathrm{APS}]_{\mathrm{aq}}$ is 0.74 or 0.71 (corrected on the water-solubility of AIBN) (Tables I and II). In the latter case the generated initiating radicals in the each phase initiate the polymerization and the difference in the polymerization rates is much closer to the experimental behavior. This approach, however, requires the transformation of the hydrophobic (derived from AIBN) primary radicals to less hydrophobic oligomeric radicals and the hydrophilic (derived from APS) radicals to the less hydrophilic oligomeric radicals before they enter the hydrophilic polymer particles and exit from the hydrophilic particles. Figure 7

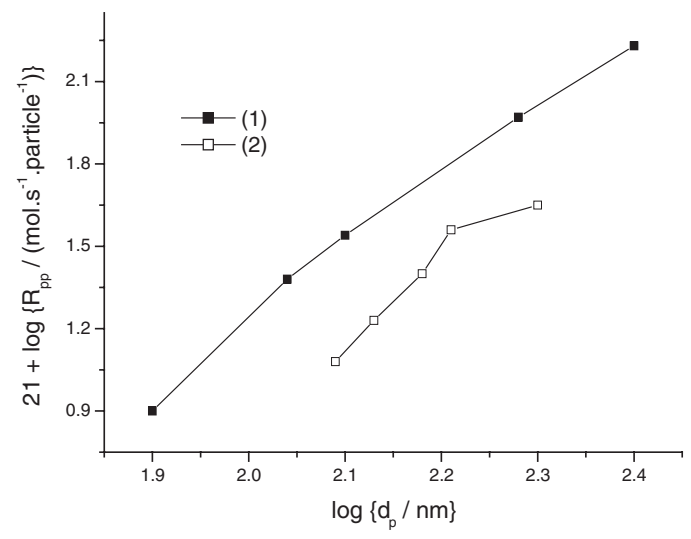

Figure 7. Variation of the polymerization rate per particle in the inverse emulsion polymerization of AAm with the particle size and initiator type. (1): APS, (2): AIBN. Other conditions see in the legends to Figures 2 and 5 and Tables I and II.

shows that the polymerization rate per particle $\left(R_{\mathrm{pp}}\right)$ proportionally increases with increasing the particle size and the increase is similar for both initiators. This dependence is the result of the two opposing processes; 1) the radical rate efficiency (proportional to the particle size) and 2) the radical desorption from particles (operative for the small particles). The $R_{\mathrm{pp}}$ 's for the same size are larger for APS. According to the ratio $[\mathrm{AIBN}]_{\mathrm{CH}} /[\mathrm{APS}]_{\mathrm{aq}}=0.74$ or 0.71 the faster APSinitiated polymerization is caused to the higher concentration of (oligomeric) radicals derived from APS at the reaction loci (Tables I and II). The spectroscopic measurements show that $c a .3 \%$ of the initial AIBN was transferred from the oil phase into the aqueous/ monomer phase. This is in a good agreement with $5 \%$ and $6.9 \%$ of the initial AIBN transferred from the oil to aqueous phase obtained for the direct emulsion systems. ${ }^{10,12}$ This indicates that the small amount of AIBN cannot influence the polymerization process.

According to the above proposed mechanism of the radical formation the reverse behavior observed in the $\mathrm{o} / \mathrm{w}$ emulsion polymerization the APS-initiated polymerization (the decomposition of APS in the continuous phase) is faster than the AIBN-initiated polymerization (the decomposition of AIBN in the disperse phase $\left.)^{31}\right\}$ can be explained as follows: the decomposition of AIBN and the addition of one or more monomer units to the primary radicals in the disperse phase forms more hydrophobic oligomeric radicals unable to exit (or with the depressed exit) into the continuous phase and take part in the further polymerization.

The dependence of the molecular weight of polyacrylamide on the emulsifier concentration for both initiators is listed in Figure 8. The molecular weight decreases with increasing the emulsifier (particle) concentration and the decrease is much more pro- 


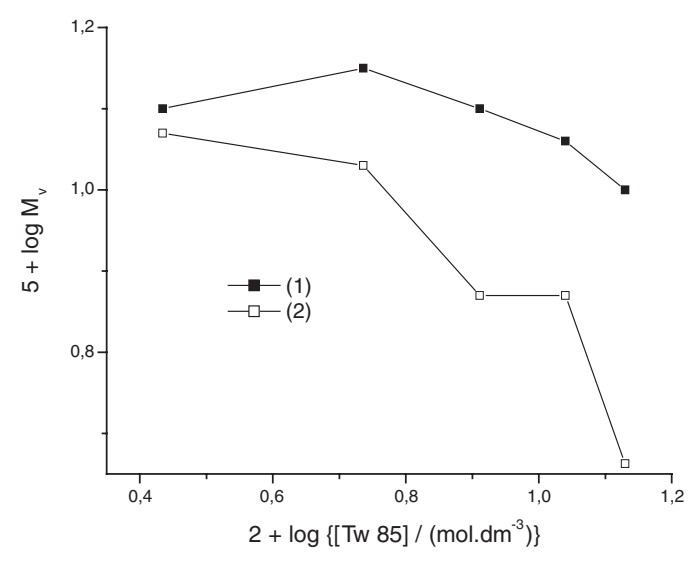

Figure 8. Variation of the molecular weight of polyacrylamide (corresponding to the final conversion) in the inverse emulsion polymerization of AAm with the Tw 85 concentration and initiator type. (1): APS, (2): AIBN. Other conditions see in the legends to Tables I and II.

nounced at high emulsifier concentrations. The similar dependence shows the polymerization rate per particle $\left(R_{\mathrm{pp}}\right) v s$. the emulsifier concentration, that is, the $R_{\mathrm{pp}}$ decreases with increasing the emulsifier concentration and the decrease is much more pronounced at high emulsifier concentrations (Figure 4). Figures 4 and 8 show that the molecular weight of polymer is proportional to the rate of polymerization, that is, the larger the polymerization rate the larger the molecular weigh of polymer. This behavior supports the compartmentalization of reaction loci and excludes the kinetics of homogeneous polymerization. The decreased concentration of monomer at the reaction loci and the chain transfer to emulsifier are responsible for the decrease in the both the polymerization rate per particle and the molecular weight of polymer especially at high emulsifier concentrations.

\section{The Fate of Radicals}

According to the micellar model the initiation of emulsion polymerization is a two-step process. The first step starts in the continuous phase by the formation of primary radicals by the decomposition of the initiator. The reaction of primary radicals with monomer (soluble in the continuous phase) leads to the formation of oligomeric radicals. The second step occurs by the entry of oligomeric radicals into the monomer swollen micelles or polymer particles. The oligomeric radicals enter the polymer particles when they reach the hydrophobicity (or hydrophilicity) similar to that for the polymer particles. According to this assumption the hydrophobic primary radicals (derived from AIBN) do not enter the hydrophilic polymer particles. The continuous-phase polymerization of AAm, however, can generate the oligomer radicals with higher hydrophilicity or with the surface activity able to enter the hydrophilic polymer particles. This indicates that the models developed for the $\mathrm{o} / \mathrm{w}$ emulsion polymerization of hydrophobic monomers such as styrene can be applied for the inverse emulsion polymerization of AAm initiated by AIBN. At the steady state, the overall rate of radical entry into particles $\rho_{\text {ovarall }}$ is expressed by

$$
\rho_{\text {ovarall }}=R_{\text {prod,c }}+R_{\text {des }}-R_{\text {ter, } \mathrm{c}}
$$

where $R_{\text {prod,c }}$ is the rate of radical production in the continuous phase, $R_{\text {des }}$ the rate of radical desorption from polymer particles and $R_{\mathrm{ter}, \mathrm{c}}$ and the rate of radical termination in the continuous phase. For the APSinitiated inverse emulsion polymerization of AAm the steady state somewhat differs from the model (eq 1) and therefore the micellar model should be somewhat modified before it is used to estimate the kinetic parameters such as radical entry and exit rate coefficients.

The average particle radical number $(\bar{n})$ estimated from the rates of polymerization divided by the number of particles and the appropriate constants decreases with increasing the particle concentration or decreasing the particle size (Tables I and II). The estimated values of $\bar{n}$ are much below 0.5 for both the APS- and AIBN-initiated polymerizations and the $\bar{n}$ is somewhat lower in the AIBN-initiated polymerizations. Generally, the very low $\bar{n}$ values are discussed in terms of the (degradative) chain transfer, the desorption of monomeric radicals from the polymer particles and the pseudo-bulk kinetics.

The original approximate equation for (monomeric) radical desorption from the polymer particles into the continuous proposed by Nomura et al. ${ }^{13}$ under the stationary steady state (eq 1 ) is

$$
k_{\mathrm{des}, N}=\left(k_{\mathrm{mf}} / k_{\mathrm{p}}\right)\left(3 D_{\mathrm{c}} \delta / m_{\mathrm{d}} r^{2}\right)
$$

where

$$
\delta=\left(1+D_{\mathrm{c}} / m_{\mathrm{d}} D_{\mathrm{p}}\right)^{-1}
$$

Here, $r$ is the particle radius, $m_{\mathrm{d}}$ the partition coefficient defined by $\left[M_{\mathrm{p}}\right]=m_{\mathrm{d}}\left[M_{\mathrm{c}}\right]\left(M_{\mathrm{p}}\right.$ : monomeric radicals in the particle phase and $M_{\mathrm{c}}$ monomeric radicals in the continuous phase) and $D_{\mathrm{c}}$ and $D_{\mathrm{p}}$ the diffusion coefficient of monomeric radicals in the continuous and particle phases, respectively. When $m_{\mathrm{d}} D_{\mathrm{p}} \gg D_{\mathrm{c}}$, $k_{\text {des }, N}$ is expressed by eq 2 , but when $m_{\mathrm{d}} D_{\mathrm{p}} \ll D_{\mathrm{w}}$, the expression for $k_{\mathrm{des}}\left(\right.$ or $\left.k_{\mathrm{des}, N}\right)$ changes to eq 3 .

$$
k_{\text {des }, N}=\left(k_{\mathrm{mf}} / k_{\mathrm{p}}\right)\left(3 D_{\mathrm{p}} / r^{2}\right)
$$

The specific desorption rate constant $k_{\text {des }}\left(\mathrm{s}^{-1}\right)$ is related to $k_{\mathrm{des}}^{\prime}\left(\mathrm{cm}^{2} \mathrm{~s}^{-1}\right)$ by the following equations: ${ }^{13,32}$

$$
k_{\mathrm{des}} a_{\mathrm{p}} / v_{\mathrm{p}}=k_{\mathrm{des}}^{\prime} /\left[(\pi / 6)^{2 / 3} d_{\mathrm{p}}^{2}\right]
$$

where $d_{\mathrm{p}}$ is the diameter of a monomer-swollen parti- 
cle, $v_{\mathrm{p}}$ the volume of monomer-swollen particles, $a_{\mathrm{p}}$ the surface area of a monomer-swollen particle. The values of $d_{\mathrm{p}}$ and $\rho_{\mathrm{m}}$ were used to calculate $v_{\mathrm{p}}$ and $a_{\mathrm{p}}$ by a simple mass balance. The parameter $k_{\mathrm{des}}^{\prime}$ is independent of particle size and, therefore, it is reasonable to compare $k_{\mathrm{des}}^{\prime}$ with the literature.

According to our best knowledge there are no data on the desorption of radicals in the inverse AAm emulsion polymerization. The solubility of styrene in the continuous (aqueous) phase (the o/w emulsion) is very low and comparable with the solubility of AAm in the continuous (organic) phase (the w/o emulsion). For the similar (restricted) solubilities of styrene and AAm in the continuous phases (styrene in water and AAm in toluene), particle sizes and monomer chain transfer constants ( $c a .0 .1-0.3$ for styrene $^{18,25}$ and $0.4-0.6$ for $\mathrm{AAm}^{20-22}$ ) the Nomura model $\left(N_{\text {model }}\right)$ estimates the similar $k_{\text {des }, N}^{\prime}$ values for both monomers. The $k_{\mathrm{des}, N}^{\prime}$ 's for the o/w emulsion polymerization of styrene initiated by the water-soluble initiator (APS dissolved in the continuous phase) at $60^{\circ} \mathrm{C}$ are in the range of $10^{-14}-10^{-12} \mathrm{~cm}^{2} \mathrm{~s}^{-1} .33,34$ The $k_{\mathrm{des}, N}^{\prime}$ 's for the inverse emulsion polymerization of AAm initiated by AIBN (dissolved in the continuous phase) are estimated to be $c a \cdot 10^{-12} \mathrm{~cm}^{2} \mathrm{~s}^{-1}$ (Table IV), that is, $k_{\mathrm{des}, N}^{\prime}$ 's for the inverse polymerization of AAm reach the upper limit of the $k_{\mathrm{des}, N}^{\prime}$ 's for the emulsion polymerization of styrene. The $k_{\mathrm{des}}^{\prime}$ 's ca. $10^{-13}-10^{-12} \mathrm{~cm}^{2} \mathrm{~s}^{-1}$ were reported together with the $\bar{n}$ below $0.5(0.2-0.4$, the emulsion polymerization of styrene).$^{34}$ The $k_{\mathrm{des}, N}$ 's are similar for both the direct emulsion polymerization of styrene and inverse emulsion polymerization of AAm but their $\bar{n}$ values strongly differ. The $k_{\text {des }, N}^{\prime}$ 's do not change with the partition coefficient $m_{\mathrm{d}}$ (the values in the brackets for the run with $[\mathrm{Tw} 85]=0.0272 \mathrm{~mol} \mathrm{dm}^{3}$, Table IV) which favors the desorption model given by eq 3 . The increased diffusion rate of radicals abruptly

Table IV. Variation of desorption rate coefficient in the inverse emulsion polymerization of AAm initiated by AIBN with the Tw 85 concentration $^{\mathrm{a}}$

\begin{tabular}{ccl}
\hline $\begin{array}{c}\text { Tw 85] } \\
\times 10^{2}\end{array}$ & $\begin{array}{c}k_{\text {des }, N}^{\prime} \\
\times 10^{13} \\
\left(\mathrm{~mol} \mathrm{dm}^{-3}\right)\end{array}$ & $\begin{array}{c}k_{\text {des }, N} \\
\times 10^{2} \\
\left(\mathrm{~cm}^{2} / \mathrm{s}\right)\end{array}$ \\
\hline 2.72 & $13.7(13.7)$ & $0.53(0.54)$ \\
5.44 & 13.7 & 0.8 \\
8.16 & $13.7(1370)$ & $0.91(91)$ \\
10.88 & 13.7 & 1.2 \\
13.6 & 13.7 & 1.4 \\
\hline
\end{tabular}

a) For estimation of $k_{\text {des }}^{\prime}$ were taken data from Tables II and III $\left(m_{\mathrm{d}}=0.093\right)$. The values of $k_{\text {des }, N}^{\prime}$ in brackets are estimated for $m_{\mathrm{d}}=0.0016\left([\mathrm{Tw} 85]=2.72 \times 10^{-2} \mathrm{moldm}^{-3}\right)$ and $\quad D_{\mathrm{p}}=2.933 \times 10^{-10} \mathrm{~cm}^{2} / \mathrm{s} \quad\left([\right.$ Tw 85$]=8.16 \times 10^{-2}$ $\mathrm{mol} \mathrm{dm} \mathrm{m}^{-3}$ ). increases the value of $k_{\mathrm{des}, N}^{\prime}$ (the values in the brackets for the run $[\mathrm{Tw} 85]=0.0816 \mathrm{~mol} \mathrm{dm}^{3}$, Table IV). This can be taken as an indication that the diluted polymer solution (the water-swollen polymer particles) could increase the exit of monomeric radicals from the polymer particles.

The dependence of the desorption rate of radicals on the rate of polymerization, the number of radicals per particle and the number of particles is modeled by the O'Toole approach. ${ }^{35}$ The experimental values of $d_{\mathrm{p}}, N_{\mathrm{p}}$ and $\bar{n}$ were used to estimate the exit rate of radicals from the polymer particles through the iterative approach comparing the experimental with theoretical $\bar{n}$ values: ${ }^{35}$

$$
\bar{n}_{\text {theor }}=a^{\prime} / 4\left[I_{m}\left(a^{\prime}\right) / I_{m-1}\left(a^{\prime}\right)\right]
$$

where $a^{\prime}=(8 a)^{0.5}, I_{m}$ and $I_{m-1}$ are the Bessel functions of the first kind of order $m$ and $m-1$, respectively. The kinetic parameters $a=\rho_{\mathrm{a}} v_{\mathrm{p}} / k_{\mathrm{t}} N_{\mathrm{p}}$ and $m=$ $k_{\text {des }} a_{\mathrm{p}} / k_{\mathrm{t}}$ are the dimensionless parameters related to adsorption of free radicals by polymer particles and desorption of radicals out of the particles, respectively. The symbol $\rho_{\mathrm{a}}$ is the absorption rate of radicals by the particles and $k_{\mathrm{t}}$ is the termination rate constant in the particle. Ugelstad and coworkers ${ }^{14}$ took into account the termination reaction in the aqueous (continuous) phase and reabsorption of desorbed radicals by the particles and developed the following relationship:

$$
a=a_{1}+m \bar{n}-Y a^{2}
$$

where $a_{1}=\rho_{\mathrm{i}} v_{\mathrm{p}} / k_{\mathrm{t}} N_{\mathrm{p}}$ and $2 N_{\mathrm{a}} k_{\mathrm{tw}} k_{\mathrm{t}} / k_{\mathrm{c}}^{2} v_{\mathrm{p}} N_{\mathrm{p}}$ are the dimensionless groups related to the generation of initiator radicals in water and termination of radicals in the aqueous phase, respectively. The parameters $k_{\mathrm{tw}}$ and $k_{\mathrm{c}}$ represent the termination rate constant in the aqueous (continuous) phase and rate constant for the capture of radicals by the particles, respectively. The rate of initiation $\left(\rho_{\mathrm{i}}\right)$ is given by

$$
\left.\rho_{\mathrm{i}}=2 f k_{\mathrm{d}} \quad \text { [initiator }\right]
$$

where $f$ is the initiator efficiency, $k_{\mathrm{d}}$ the decomposition rate constant of initiator and [initiator] the molar concentration.

With the assumption that termination in the continuous phase is not important (i.e., $Y=0$ ), $a$ (or $\rho_{\mathrm{a}}$ ) and $m$ (or $k_{\mathrm{des}, T}$ ) can be calculated as,

1) Guess a value for $m$

2) Compute $a$ according to eq 6

3) Compute $\bar{n}$ by eq 5

4) If calculated $n$ is equal to the experimental value, accept the theoretical values of $a$ and $m$. If not, go back to step 1 .

5) Compute $\rho_{\mathrm{a}}=a k_{\mathrm{t}} N_{\mathrm{p}} / v_{\mathrm{p}}$ and $k_{\text {des }}=m k_{\mathrm{t}} / a_{\mathrm{p}}$.

Ugelstad et al. $^{32}$ solved the simultaneous eqs 5 
Table V. Variation of kinetic parameters in the inverse emulsion polymerization of AAm initiated by AIBN with the Tw 85 concentration $^{\text {a }}$

\begin{tabular}{ccccccc}
\hline $\begin{array}{c}{\left[\mathrm{Tw} \mathrm{85]} \times 10^{2}\right.} \\
\left(\mathrm{mol} \mathrm{dm}^{-3}\right)\end{array}$ & $a \times 10^{4}$ & $m$ & $k_{\text {des }} / \mathrm{s}$ & $\begin{array}{c}k_{\mathrm{des}}^{\prime} \times 10^{9} \\
\left(\mathrm{~cm}^{2} / \mathrm{s}\right)\end{array}$ & $\begin{array}{c}\rho_{\mathrm{a}} \\
\left(\mathrm{mol} \mathrm{dm}^{-3} \mathrm{~s}^{-1}\right)\end{array}$ & $\rho_{\mathrm{a}} / \rho_{\mathrm{i}}$ \\
\hline 2.72 & 25.0 & 0.84 & 1650 & 2.2 & 16.6 & 13.5 \\
5.44 & 7.0 & 0.363 & 1340 & 2.3 & 5.7 & 16.7 \\
8.16 & 4.74 & 0.336 & 1480 & 2.2 & 4.5 & 19.1 \\
10.88 & 2.27 & 0.562 & 3664 & 4.2 & 4.0 & 36.7 \\
13.6 & 1.4 & 0.563 & 4635 & 4.6 & 3.1 & 45.4 \\
\hline
\end{tabular}

${ }^{\mathrm{a}}$ For other conditions see the legend to Table I.

and 6 for $\bar{n}_{\text {theor }}$ and plotted the calculated $\bar{n}_{\text {theor }}$ 's against the value of $a_{1}$ at fixed value of $Y$, varying the value of $m$ as a parameter. For the emulsion polymerization of styrene the value of $m$ is about $10^{-4}$ or smaller. The values of $m$ for the inverse emulsion polymerization of AAm is $c a$. by one order in the magnitude larger ( $c a$. about $10^{-3}$, Table V) which indicates a increased desorption of monomeric radicals from the polymer particles. The values of $m$ and $a$ (their position in the $\bar{n}_{\text {theor }} v s . a_{1} \operatorname{plot}^{32}$ ) suggest the desorption of monomeric radicals from the particles and the compartmentalized nature of the reaction loci (the suspension polymerization mechanism is excluded).

The $k_{\text {des }, T}^{\prime}($ Table $\mathrm{V})$ values for the desorption of monomeric radicals from particles are by several orders in the magnitude larger than the corresponding $k_{\mathrm{des}, N}^{\prime}$ 's (Table IV). The very low concentration of radicals in the particles and the very large $k_{\mathrm{des}, T}^{\prime}$ 's are in the strong support of the robust desorption of radicals. The model of desorption of radicals has the following steps: ${ }^{13} 1$ ) transfer of free radical activity to monomer, 2) diffusion of the resulting monomeric radical, or small oligomeric, free radical to the particle surface, and then, if propagation beyond a certain degree of polymerization has not occurred, 3) diffusion of the free radical species away from the particle through the aqueous phase. The rate limiting step for the exit of radicals from present relatively large particles is the diffusion of monomeric radicals to the particle surface. The surface active AAm monomer is known to concentrate in the interfacial layer due to which increases the contribution of the polymerization in the upper spheres of polymer particles. ${ }^{2}$ The chain transfer to emulsifier and monomer and the exit of both the monomeric and emulsifier radicals into the organic continuous phase govern the polymerization process. The $k_{\text {des }, T}^{\prime}$ 's were somewhat larger at the high emulsifier concentration. Furthermore, the high-packed interfacial layer is formed at high emulsifier concentrations. This is accompanied with the abrupt increase in the surface area of the droplets which favors the desorption of radicals. ${ }^{36}$

The strong desorption of monomeric radicals indi- cates that the whole radical concentration in the continuous phase consists of two parts; the primary radicals derived from AIBN and monomeric radicals desorbed from particles. Table $\mathrm{V}$ shows that the ratio $\rho_{\mathrm{a}} / \rho_{\mathrm{i}}$ is much above 1 and it decreases with increasing the number of particles or the emulsifier concentration. The behavior $\left(\rho_{\mathrm{a}} / \rho_{\mathrm{i}} \gg 1\right)$ can be discussed in terms of the efficient entry of radicals into particles and/or the contribution of continuous phase termination. The decreased molecular weights of PAAm with increasing the emulsifier concentration are in favor of the increased termination. The continuous decrease of the $\rho_{\mathrm{a}} / \rho_{\mathrm{i}}$ parallels the decrease in the particle size. The smaller the particle size the lower the radical entry rate $\left(\rho_{\mathrm{a}}\right)$. The increased fraction of emulsifier in the interfacial layer indicates the decrease in the interaction (reaction) of entering radicals with the surface active monomer (AAm). The fail in the propagation leads to the desorption of monomeric radicals.

Table VI shows that the estimated kinetic parameters for the APS-initiated polymerization are very similar to those obtained for the AIBN-initiated one (Table V). This indicates that the mechanism of polymerization and the steady-state for the radicals are similar for both initiators. The $R_{\text {prod,c }}$ parameter in the eq 1 , thus, is more complex and includes the formation of primary and oligomer radicals and the partitioning of radicals with the initiator end group and one or more added monomer units between oil and water phases (for AIBN). In the case of AIBN the continuous phase contains the primary and oligomeric radicals derived from initiator and desorbed (monomeric and emulsifier) radicals. In the case of APS the continuous phase contains oligomeric radicals (due to the partitioning events) and desorbed radicals.

\section{CONCLUSIONS}

The inversion emulsion polymerizations initiated by both APS and AIBN initiators are very fast and reach the final conversion during several minutes. The shapes of conversion curves and polymerization rate versus conversion curves are very similar for both 
Table VI. Variation of kinetic parameters in the inverse emulsion polymerization of AAm initiated by APS with the Tw 85 concentration $^{\text {a }}$

\begin{tabular}{cclcccr}
\hline $\begin{array}{c}{\left[\mathrm{Tw} \mathrm{85]} \times 10^{2}\right.} \\
\left(\mathrm{mol} \mathrm{dm}^{-3}\right)\end{array}$ & $a \times 10^{4}$ & $m$ & $k_{\mathrm{des}} / \mathrm{s}$ & $\begin{array}{c}k_{\text {des }}^{\prime} \times 10^{9} \\
\left(\mathrm{~cm}^{2} / \mathrm{s}\right)\end{array}$ & $\begin{array}{c}\rho_{\mathrm{a}} \\
\left(\mathrm{mol} \mathrm{dm}^{-3} \mathrm{~s}^{-1}\right)\end{array}$ & $\rho_{\mathrm{a}} / \rho_{\mathrm{i}}$ \\
\hline 2.72 & 45.0 & 0.087 & 73.4 & 2.7 & 12.2 & 1.9 \\
5.44 & 7.0 & 0.05 & 109.0 & 2.6 & 2.9 & 2.9 \\
8.16 & 0.64 & 0.0333 & 238 & 2.6 & 0.58 & 6.4 \\
10.88 & 0.234 & 0.0282 & 331 & 2.6 & 0.31 & 9.3 \\
13.6 & 0.035 & 0.0355 & 1080 & 4.5 & 0.13 & 25.7 \\
\hline
\end{tabular}

${ }^{\mathrm{a}}$ For other conditions see the legend to Table II.

initiators. The rate of polymerization $v s$. conversion is described by a curve with three rate intervals. For both initiators the polymerization rate per particle is proportional to the particle size and decreases with increasing the emulsifier concentration and the decrease is much more pronounced at the high emulsifier concentrations. The similar kinetic dependences for both initiators were discussed in terms of the formation of radicals with the similar hydrophobicity (or hydrophilicity), diffusivity via the reaction system and partitioning between the oil and water phases. The primary radicals with an initiator group $\mathrm{SO}_{4}{ }^{\bullet-}$ or 2-cyanoisopropyl concentrate in water or cyclohexane. The polymerization generates the radicals with an initiator group $\mathrm{SO}_{4}{ }^{\bullet-}$ and 2-cyanoisopropyl and one or more added monomer units which are supposed to have similar partitioning coefficients between the water and oil phases. The average number of radicals per particle $(\bar{n})$ was observed to be much below 0.5 . The very low radical concentration in particles was attributed to the strong desorption of low molecular weight radicals (monomeric, emulsifier and radicals with the initiator end group and one or more added monomer units). The desorption rate constant $k_{\mathrm{des}}^{\prime}$ estimated by the Nomura model and Ugelstad approach indicates the desorption of radicals and the desorption rate is much more pronounced in the Ugelstad approach. The strong radical desorption was attributed to the desorption of both monomeric and emulsifier radicals. The polymerization of surface active AAm is connected with the increased chain transfer to the emulsifier and exit of monomeric and emulsifier radicals. The ratio $\rho_{\mathrm{a}} / \rho_{\mathrm{i}} \gg 1$ was attributed to the increased entry of radicals into particles. The continuous decrease of the $\rho_{\mathrm{a}} / \rho_{\mathrm{i}}$ parallels the decrease in the particle size. The increased fraction of emulsifier in the interfacial layer is connected with the depressed interaction (reaction) of entering radicals with the surface active monomer (AAm).

Acknowledgment. This research is supported by the Slovak Grand Agency (VEGA) through the grant number 2/4008/04. The author thanks Prof. M.
Nomura for his comments concerning of using his model in the inverse emulsion polymerization and Mrs. K. Cínová for her assistance with the experimental work. We appreciate the help rendered by Prof. C. S. Chern, Department of Chemical Engineering, National Taiwan University of Science and Technology, Taipei, Taiwan, in the computational (modeling) work.

\section{REFERENCES}

1. J. W. Vanderhoff, E. B. Brandford, H. L. Tarkowski, J. B. Shaffer, and R. M. Wiley, Adv. Chem. Ser., 34, 32 (1962).

2. F. Candau, "Polymerization in Microemulsions," in "Polymerization in Organized Media," C. M. Paleos, Ed., Gordon and Breach Publishers, Philadelphia, PA, 1992, p 215.

3. V. Vašková, V. Juraničová, and J. Bartoň, J. Makromol. Chem., 191, 717 (1990).

4. J. Barton, Prog. Polym. Sci., 21, 399 (1996).

5. K. Landfester, M. Willert, and M. Antonietti, Macromolecules, 33, 2370 (2000).

6. K. O. Kobyakova, V. F. Gromov, and E. N. Teleshov, Polym. Sci., 35, 151 (1993).

7. J. H. Barajas and D. J. Hunkeler, Polymer, 38, 437, 5623 (1997).

8. P. Y. Jiang, Z. C. Zhang, and M. W. Zhang, Radiat. Phys. Chem., 45, 825 (1995).

9. J. W. Breitenbach and H. Edelhauser, Makromol. Chem., 44-46, 196 (1961).

10. J. Barton and I. Capek, "Radical Polymerization in Disperse Systems," T. J. Kemp, Ed., Harwood, Chichester, U.K., 1994.

11. M. Nomura and K. Fujita, Makromol. Chem., Rapid Commun., 10, 581 (1989).

12. J. A. Alduncin, J. Forcada, M. J. Barandiaran, and J. M. Asua, J. Polym. Sci., Polym. Chem. Ed., 29, 1265 (1991).

13. M. Nomura, H. Harada, K. Nakagawara, W. Eguchi, and S. Nagata, J. Chem. Eng. Jpn., 4, 160 (1971).

14. J. Ugelstad and P. C. Mork, Br. Polym. J., 2, 31 (1970).

15. P. Potisk and I. Capek, Angew. Makromol. Chem., 222, 125 (1994).

16. W. Soltan, Makromol. Chem., 14, 169 (1954).

17. I. Capek and S. Janickova, Chem. Pap., in press.

18. J. Brandrup and E. H. Immergut, "Polymer Handbook, 4th ed.," Wiley-Interscience, New York, N.Y., 1998. 
19. N. Grassie and E. Vance, Trans. Faraday Soc., 52, 727 (1956).

20. D. J. Currie, F. S. Dainton, and W. S. Watt: Polymer, 6, 451 (1965).

21. T. Ishige and A. E. Hamielec, J. Appl. Polym. Sci., 17, 1479 (1973).

22. C. J. Kim and A. C. Hamielec, Polymer, 25, 845 (1984).

23. H. Kawaguchi, Y. Sugi, and Y. Ohtsuka, J. Appl. Polym. Sci., 26, 1649 (1981).

24. D. C. MacWilliams, "Acrylamide, and Other Alpha, Beta Unsaturated Amides," in "Functional Polymers, Their Preparation, Polymerization, and Application," Vol. 1, R. H. Yocum and E. B. Nuquist, Ed., Marcel Dekker, New York, N.Y., 1993, p 12.

25. J. Gao and A. Penlidis, Prog. Polym. Sci., 27, 403 (2002).

26. V. Juranicova and I. Capek, Macromol. Symp., 179, 275 (2002).

27. W. V. Smith and R. H. Ewart, J. Chem. Phys., 16, 592
(1948).

28. M. T. Carver, U. Dreyer, R. Kmoesel, F. Candau, and R. M. Fitch, J. Polym. Sci., Polym. Chem. Ed., 27, 2161 (1989).

29. I. Capek and P. Potisk, J. Polym. Sci., Polym. Chem. Ed., 33, 1675 (1995).

30. R. M. Fitch and C. H. Tsai, J. Polym. Sci., Polym. Lett. Ed., 8, 703 (1970).

31. I. Capek, Adv. Colloid Interface Sci., 91, 295 (2001).

32. J. Ugelstad, P. C. Mork, and J. O. Aassen, J. Polym. Sci., 5, 2281 (1967).

33. H. C. Lee and G. W. Poehlein, Polym. Process Eng., 5, 37 (1987).

34. C. S. Chern, S. Z. Lin, and T. J. Hsu, Polym. J., 31, 516 (1999).

35. J. I. Toole, J. Appl. Polym. Sci., 9, 1291 (1965).

36. M. Harada, M. Nomura, W. Eguchi, and S. Nagata, J. Chem. Eng. Jpn., 4, 54 (1991). 